\title{
Stress Biology in Microalgae Depicts Molecular Insights for Simultaneous Production of Lipids and High Value Precursors
}

\author{
Ashwani K Rai ${ }^{1}$, Asha A Nesamma ${ }^{2 *}$ and Pannaga P Jutur ${ }^{2}$ \\ ${ }^{1}$ Department of Biological Sciences, Louisiana State University, USA \\ ${ }^{2}$ International Centre for Genetic Engineering and Biotechnology (ICGEB), India
}

Submission: August 31, 2017; Published: October 23, 2017

*Corresponding author: Asha A Nesamma, Scientist, Omics of Algae Group, Integrative Biology, DBT-ICGEB Centre for Advanced Bioenergy Research, International Centre for Genetic Engineering and Biotechnology (ICGEB), Aruna Asaf Ali Marg, New Delhi 110067, India, Tel: +911126741358; Fax: +911126742316; Email: asha@icgeb.res.in

\begin{abstract}
Microalgae are the promising resource of renewable energy to replace decreasing oil reserves as a source of lipids and high value precursors for biofuels. These algal oils are rich in the triacylglycerols (TAGs) that serve as material for conversion to biofuels. Studies on the biosynthetic pathways and rate limiting steps of triacylglycerols formation in microalgae are limited. Hence, microalgae have been considered as potential feedstock to produce higher biomass and lipid content that is more suitable for biofuel production than traditional oleaginous crop plants, thus seems to be a niche for accumulating energy reserves to produce next generation renewable such as biofuels and high-value chemicals, an essential alternative for diminishing fossil fuels. Evaluation of growth and lipid profiles of oleaginous freshwater microalgae under nutrient deprivation will be the method to identify best industrial strain for production of biofuel and high value precursors at the commercial level. In the present study, we have evaluated two freshwater microalgae strains i.e. Chlorella sp. and Tetraselmis sp. to find out their metabolic responses on growth and lipid profiles under different nutrient-limited (nitrogen, phosphorous and/or sulfur) conditions. Our results demonstrate that all these strains showed severe growth hampering by stress phenomenon under nutrient deprivation, with few exceptions. Henceforth, depicting molecular insights triggered by nutrient stress biology in these microalgae demonstrated primarily increased TAG content ( up to 17-18 mg $\mathrm{L}^{-1} \mathrm{D}^{-1}$ ) in both freshwater strains. In conclusion, the simultaneous production of lipids and high-value precursors among these oleaginous strains will be further characterized, which may be a critical step towards making algae-derived biofuels economically competitive for industrial production.
\end{abstract}

Keywords: Biomass; Lipid; Microalgae; Oleaginous; Triacylglycerols

Abbreviations: TAGs: Triacylglycerols; FAMES: Fatty Acid Methyl Esters; GC-MS: Gas Chromatography-Mass Spectrometry; SPV: Sulfo Phospho Vanillin

\section{Introduction}

Nearly $40-50 \%$ of total global photosynthesis occurring on earth is primarily due to the presence of these algal populations [1]. Microalgae have been identified as a viable feedstock for biofuels due to their efficient capabilities to convert sunlight and $\mathrm{CO}_{2}$ to biomass (which is of great importance for the feed, fuel and chemical industries), can thrive in fresh to saline waters, and the ability to grow on non-arable lands that has led their path towards the replacement of fossil fuels with sustainable renewable biofuels pertaining to ever increasing energy demands [2].

Several oleaginous strains of microalgae have been identified which are capable to produce naturally high levels of lipids (e.g. $20-75 \%$ dry mass) in the form of triacylglycerols (TAGs), which can be converted for use as biodiesel via transesterification process. Many research findings demonstrated that stress responses in these microalgae could induce the formation of neutral lipids (triacylglycerols-TAGs, the precursors for biofuel production) under various stress conditions like starvation, temperature changes, salt concentration but the major hurdle being hindered cell growth $[3,4]$. Microalgae are considered as key players in producing oils (biofuel precursors) compared to other terrestrial crops (e.g. palm, rapeseed, and soybean) and thus have gained importance as economical feedstock with certain challenges [5]. Microalgae biofuels are considered as one of the viable alternative sources of energy as they are renewable, sustainable and environmental-friendly and reconstruction of these metabolic pathways for increasing oil content is foreseen as a niche in algal biology [6]. 
Although microalgae biofuels hold great promises, still considerable challenges exist for their commercialization. Generally, the lipid composition among microalgae varies between $20-75 \%$ (dw) depending on the species as well as the environmental conditions [7-10]. Recently, various biochemical strategies have been employed to improve lipid accumulation and biomass production [11,12]. Nutrient availability has a significant impact on growth of microalgae as well as broad effects on their lipid and fatty acid composition [3,13-15]. Henceforth, nutrient deprivation is one of the most widely used and applied lipid induction techniques followed in enhancing microalgal lipid and high value precursor production where they tend to change their metabolic profiles and biochemical composition. Producing these value-added biorenewable in addition to biofuels, fatty acid methyl esters (FAMEs), and lipids has the potential to improve microalgae-based biorefineries by employing either the autotrophic or the heterotrophic mode, which could be an offshoot of biotechnology [16].

In the present study, we focussed on screening and characterization of two different freshwater oleaginous microalgal strains depending on their growth (biomass) and lipid profiles under different nutrient limitations, viz. nitrogen, phosphorous and sulphur for biofuel production and the overview of this study is to assess the effect of nutrient deprivation i.e., stress biology for simultaneous production of microalgal lipids and high value precursors.

\section{Materials and Methods}

\section{Strains and culture conditions}

Two freshwater microalgal strains were used in this study namely Chlorella sp and Tetraselmis sp procured from Institute of bioresources and Sustainable Development (IBSD), Impala, Manipur, India. Cultures were incubated under continuous illumination $\left(\sim 120 \mu \mathrm{mol} \mathrm{m} \mathrm{m}^{-2} \mathrm{~s}^{-1}\right.$ photo synthetically active radiation [PAR]) on an orbital shaker (200 rpm) at $25{ }^{\circ} \mathrm{C}$ and ambient levels of $\mathrm{CO}_{2}$. Cells were initially grown photoautotrophically to the middle of the logarithmic phase in modified BG-11 minimal medium. The composition of media is [in g L ${ }^{-1}$ ] $-\mathrm{K}_{2} \mathrm{HPO}_{4}-40 ; \mathrm{MgSO}_{4} .7 \mathrm{H}_{2} \mathrm{O}-75 ; \mathrm{CaCl}_{2} .2 \mathrm{H}_{2} \mathrm{O}$ - 36; Citric acid - 6; $\mathrm{FeC}_{6} \mathrm{H}_{5} \mathrm{O} 7 \mathrm{NH}_{4} \mathrm{OH}$ - 6; EDTA - 1; $\mathrm{Na}_{2} \mathrm{CO}_{3}-20 ; \mathrm{NaNO}_{3}-750$; and BG-11 trace metal solution $\mathrm{H}_{3} \mathrm{BO}_{3}-2.85 ; \mathrm{MnCl}_{2} .4 \mathrm{H}_{2} \mathrm{O}-1.80$; $\mathrm{ZnSO}_{4} \cdot 7 \mathrm{H}_{2} \mathrm{O}-0.20 ; \mathrm{Na}_{2} \mathrm{MoO}_{4} \cdot 2 \mathrm{H}_{2} \mathrm{O}-0.40 ; \mathrm{CuSO}_{4} \cdot 5 \mathrm{H}_{2} \mathrm{O}-0.08$; $\mathrm{Co}$ $\left.\left(\mathrm{NO}_{3}\right)_{2} .6 \mathrm{H}_{2} \mathrm{O}-0.05\right)$. These pre-cultured cells were collected by centrifugation and resuspended at a density of $6 \times 10^{5}$ cells $\mathrm{mL}^{-1}$ in regular BG-11 media or in the same media lacking nitrogen $(-N)$, phosphorus (-P), and sulfur (-S). Samples for analysis were taken immediately after suspension (Control, 0 day) and at the time intervals of 2, 4, and 6 days. Culture growth was monitored by counting cells with a haemocytometer [17]. Growth rates were calculated by the following equation.

$$
K^{\prime}=\left(\ln N_{2} / N_{1}\right) /\left(t_{2}-t_{1}\right)
$$

Where $\mathrm{N}_{1}$ and $\mathrm{N}_{2}$ equals cell count at time $1\left(\mathrm{t}_{1}\right)$ and time $2\left(t_{2}\right)$, respectively. Doubling time was also calculated once the specific growth rate was known [17].

$$
\text { Doubling time }=\ln _{2} / K^{\prime}
$$

\section{Quantification of total lipids by sulfo-phospho-vanillin (SPV) assay}

The standard lipid stock (s) were prepared using commercial canola oil (2 $\left.\mathrm{mg} \mathrm{mL}^{-1}\right)$ in chloroform, which was subsequently stored at $-20^{\circ} \mathrm{C}$ before use. Different concentrations of lipid in microfibers of standard oil solution were added in the empty tube. The tubes were incubated at $60{ }^{\circ} \mathrm{C}$ for $10 \mathrm{~min}$ to evaporate the solvent and $100 \mu \mathrm{l}$ of water was added to the lipid standard. Further sample was prepared by following SPV reaction methods. Teflon-covered glass vials were used throughout all experiments. Phospho-vanillin reagent was prepared by initially dissolving $0.6 \mathrm{~g}$ vanillin in $10 \mathrm{ml}$ absolute ethanol; $90 \mathrm{ml}$ deionised water and stirred continuously. Subsequently $400 \mathrm{ml}$ of concentrated phosphoric acid was added to the mixture and the resulting reagent was stored in the dark coloured bottle until use. To ensure high activity, fresh phospho-vanillin reagent was prepared shortly before every experiment run. For SPV reaction of the algal culture for lipid quantification, a known amount of biomass in $100 \mu$ l of water, which are either suspended in a known volume of liquid culture or harvested via centrifugation at 4,000 RPM for $5 \mathrm{~min} .2 \mathrm{ml}$ of concentrated (98\%) sulphuric acid was added to the sample and heated for $10 \mathrm{~min}$ at $100{ }^{\circ} \mathrm{C}$ and cooled for $5 \mathrm{~min}$ in ice bath. $5 \mathrm{ml}$ of freshly prepared phospho-vanillin reagent was added and the sample was incubated for $15 \mathrm{~min}$ at $37{ }^{\circ} \mathrm{C}$ incubator shaker at $200 \mathrm{rpm}$. The absorbance reading at $530 \mathrm{~nm}$ was taken in order to quantify the lipid within the sample $[18,19]$. For blank, culture extracts replaced with water $(100 \mu \mathrm{l})$, sulphuric acid $(2 \mathrm{ml})$ and $5 \mathrm{ml}$ of SPV reagent was used.

\section{FAME analysis by gas chromatography-mass spectrometry}

For fatty acid methyl esters (FAME) analysis, lipids in the microalgal cells (approximately $\sim 1 \times 10^{8}$ cells) were hydrolysed and methylesterified in $300 \mu \mathrm{l}$ of a $2 \% \mathrm{H}_{2} \mathrm{SO}_{4}$ and methanol solution for $2 \mathrm{~h}$ at $80{ }^{\circ} \mathrm{C}$. Prior to the reaction, $50 \mu \mathrm{g}$ of heptadecanoic acid (Alfa Aesar, USA) was added as internal standard. After esterification step, $300 \mu \mathrm{l}$ of $0.9 \%(\mathrm{w} / \mathrm{v}) \mathrm{NaCl}$ solution and $300 \mu \mathrm{l}$ of hexane was added and mixed thoroughly for 20s. To separate the phase, samples were centrifuged at $3,000 \mathrm{x}$ g for $3 \mathrm{~min}$. A total of $1 \mu \mathrm{l}$ of hexane layer was injected into an Agilent 6890 gas chromatograph (GC) coupled to mass spectrometer (MS) [3,20]. The running conditions for GC-MS were described by Agilent's RTL DBWax method [21].

\section{Result and Discussion}

\section{The effect of nutrient deprivation on the growth of Chlorella sp and Tetraselmis sp}

The growth of freshwater microalgae Chlorella sp. and Tetraselmis sp. subjected to nutrient replete (control) and 
medium deprived of different nutrients $(\mathrm{N}, \mathrm{P}$, and $\mathrm{S})$ in minimal BG-11 media under continuous illumination of light was shown in Figures 1A \& 1B. Both species of microalgae had initial lag phase and exponential growth phase followed by the late log phase at day 10 in all medium, although some of them were not very obvious. Previous studies on the total nutrient deprivation resulted in the largest reduction of algal growth, followed by deprivation of nitrogen, phosphate and sulfur from the medium [22]. The influence of nutrient deprivation on the microalgal growth seemed to be species-specific or have different performance in different culture conditions [23]. Deprivation of phosphorus from the medium reduced the growth rate of both Chlorella sp and Tetraselmis sp than control (Figure 1A \& 1B). However, the phosphorus deprivation significantly reduced the optical densities of Chlorella sp. and Tetraselmis sp. from day 6 to day 10 compared to the BG-11 medium (Figures $1 \mathrm{~A} \& 1 \mathrm{~B}$ ).

Nitrogen limitation of Chlorella $\mathrm{sp}$ and Tetraselmis $\mathrm{sp}$ had drastic effect on the growth with the complete nutrition deprivation. sulfur deprivation had different effect on the growth i.e. in Chlorella sp shown drastic reduction in growth whereas Tetraselmis sp significantly reduced the optical densities which varied from day 6 to day 10 compared to control. These results suggested that different microalgae respond differently to the different nutrient deprivation. Although nutrient deprivation can increase the lipid content of microalgae, it reduced the growth and biomass of the microalgae more or less, which is not desirable in the lipid production.

Table 1: *Average of three biological replicates \pm standard deviations.

\begin{tabular}{|c|c|c|c|}
\hline Species & $\begin{array}{c}\text { Biomass } \\
\text { Production }\left(\mathbf{g ~ L}^{-1}\right)\end{array}$ & $\begin{array}{c}\text { Specific Growth } \\
\text { Rate } \boldsymbol{\mu}\left(\text { day }^{-1}\right)^{*}\end{array}$ & $\begin{array}{c}\text { Doubling } \\
\text { Time(h) }\end{array}$ \\
\hline Chlorella sp & 0.98 & 0.23 & 69.6 \\
\hline Tetraselmis sp & 0.76 & 0.25 & 64.3 \\
\hline
\end{tabular}

Figure 1A

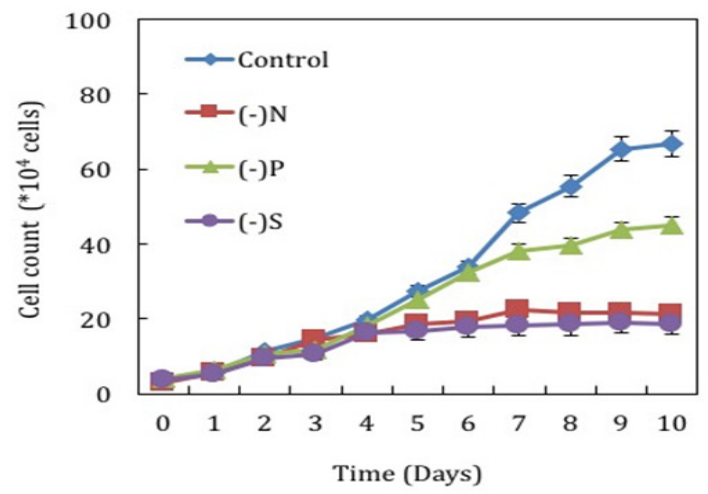

Figure 1B

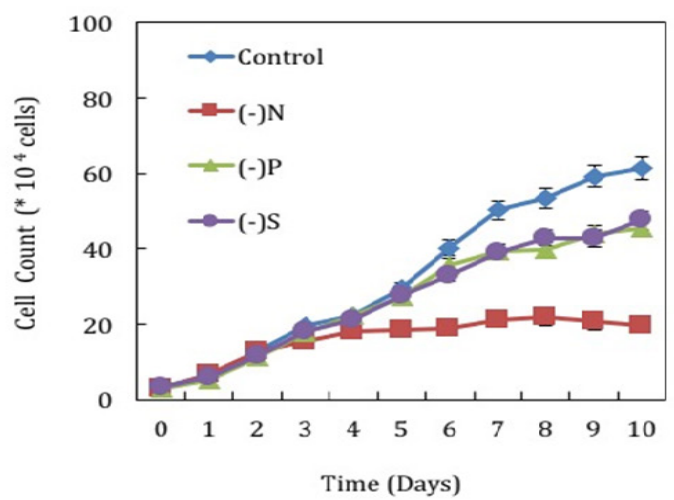

Figure 1A: Growth profiles of freshwater microalgae Chlorella sp. subjected to continuous illumination of light under nutrient replete and deplete [-N; - $\mathrm{P} ;-\mathrm{S}]$ conditions in minimal (BG-11) media.

Figure 1B: Growth profiles of freshwater microalgae Tetraselmis sp. subjected to continuous illumination of light under nutrient replete and deplete $[-\mathrm{N} ;-\mathrm{P} ;-\mathrm{S}]$ conditions in minimal (BG-11) media.

Nutrient availability is critical for cell division and intracellular metabolite cycling and once nutrients such as $\mathrm{N}$, $\mathrm{P}$, and $\mathrm{S}$ become depleted or limited in the medium, invariably, a steady decline in reproduction rate ensues. Once this occurs, the activated metabolic pathways responsible for biomass production are down-regulated and cells instead divert and deposit much of the available $C$ into lipid [24,25]. In the present study, two freshwater microalgal strains were subjected to nutrient (N, P, and S) deprivation to understand their effect on the growth and simultaneous production of lipids and high value precursors, to determine potential candidate for biofuel production. Table 1 shows the growth profiles of two freshwater microalgae Chlorella sp. and Tetraselmis sp. in controlled growth conditions. The biomass production of Chlorella sp. and Tetraselmis sp. were 0.98 and $0.76 \mathrm{gL}^{-1}$ respectively and exhibited similar specific growth rates of 0.23 and 0.25 day $^{-1}$. Studies demonstrate that under optimal growth conditions (i.e. adequate supply of nutrients including C, N, P, S and sunlight), biomass productivity can exceed $30 \mathrm{~g}$ dry weight per square meter per day with very low $(<5 \% \mathrm{w} / \mathrm{w})$ lipid content and is species dependent [26]. In principle, biomass production and lipid biosynthesis compete for photosynthetic assimilation of inorganic carbon, and a metabolic shift is required to switch from biomass synthesis to energy storage metabolism $[3,25]$. Thus, lipids are typically believed as storage reserves within the cell that enables the organism to survive adverse environmental and/or nutrient-limited conditions [27]. To analyze the growth profile changes under different nutrient deprivations such as nitrogen $(-\mathrm{N})$, phosphorus $(-\mathrm{P})$, and sulfur $(-\mathrm{S})$, these strains were grown in strict photoautotrophic conditions in minimal BG-11 media following sampling at time intervals of $24 \mathrm{~h}$. To determine the biochemical/metabolic changes under similar conditions in these microalgae, the samplings for other experiments were collected at time intervals of $0,48,96$ and $144 \mathrm{~h}$. Our results demonstrate that Chlorella sp. had severe effect on growth in nitrogen $(-\mathrm{N})$ and sulfur $(-\mathrm{S})$ depletion after $3^{\text {rd }}$ day whereas 
under phosphorus (-P) depletion decline in growth was seen after $6^{\text {th }}$ day (Figure 1A). In Tetraselmis sp. under sulfur $(-S)$ and phosphorous (-P) depletion, the growth is stunted after $7^{\text {th }}$ day while under nitrogen $(-\mathrm{N})$ depletion growth is severely inhibited by $3^{\text {rd }}$ day onwards as shown in Figure $1 \mathrm{~B}$. The effect of $\mathrm{N}, \mathrm{P}$, and $\mathrm{S}$ deprivation on growth of freshwater microalgae Chlorella sp. and Tetraselmis sp. subjected to 10 days of nutrient deprivation with continuous illumination of light in minimal BG-11 media triggered a wide range of different unique responses in these microalgae.

\section{Lipid analysis and profiling in two freshwater microalgae Chlorella sp. and Tetraselmis sp. subjected to nutrient deprivation}

Stress-induced strategies for enhancing lipid accumulation, such as nutrient depletion is the most prevalent method employed in microalgae [23,28-30]. This is primarily due to two factors:
1. Lack of requisite nutrients such as $\mathrm{N}, \mathrm{P}$, and $\mathrm{S}$ limits the cellular division forcing the organism to take advantage of alternative pathways for inorganic carbon fixation, where de novo fatty acids (in the form of TAGs) are synthesized and stored [29]

2. Photosynthesis and the electron transport chain produce ATP and NADPH as energy storage and electron carrier metabolites, respectively in eukaryotic microalgae which is consumed during biomass production [31].

Under normal growth conditions, the balanced ratio of reduced and oxidized metabolites is maintained; however under nutrient starvation, due to lack of requisite nutrients, the pool of NADP+ and ADP can become depleted [27]. Fatty acid synthesis consumes NADPH and ATP, therefore increased fatty acid synthesis replenishes the pool of required electron acceptors in the form of NADP+ and de novo fatty acids are stored as lipids [21].

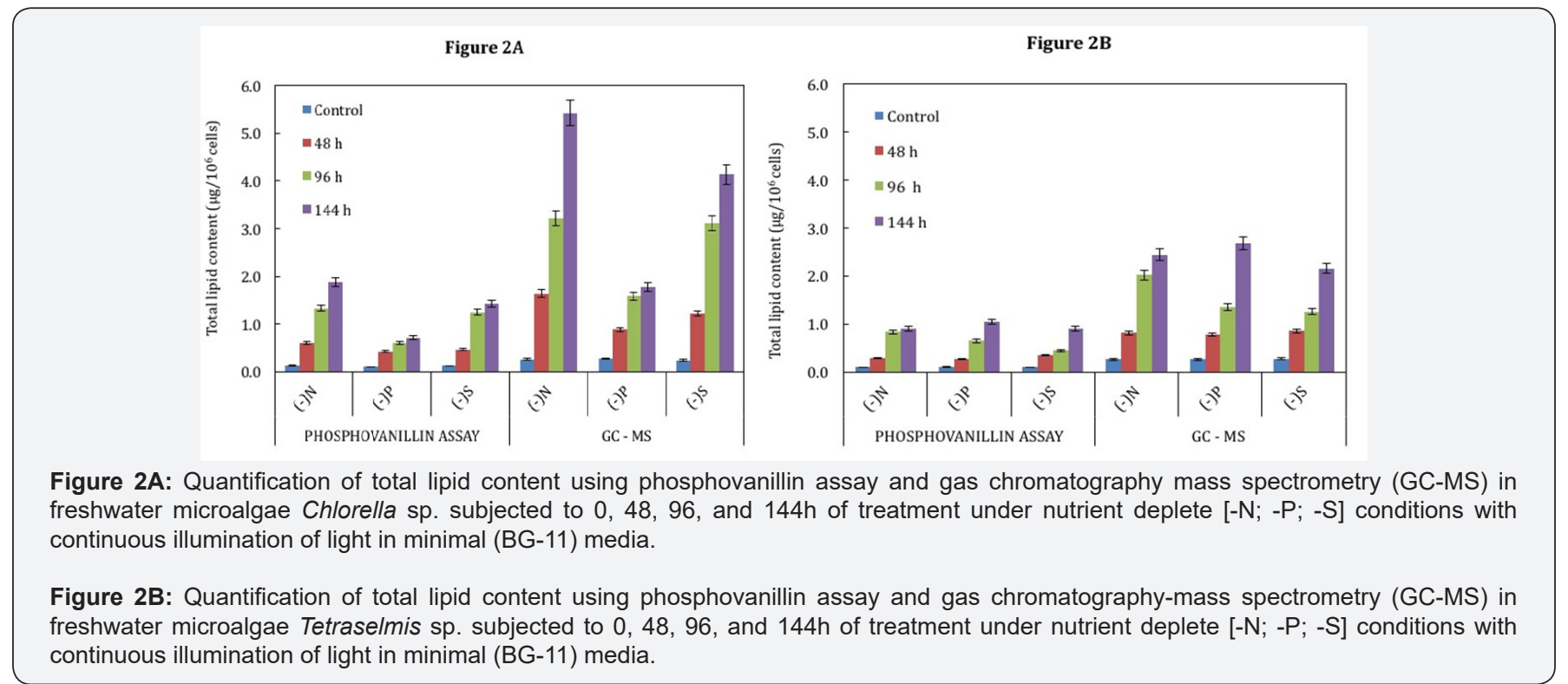

Total lipids were extracted from both the microalgal strains and analysed by phosphovanillin assay and GC-MS as described in materials and methods, to understand the formation of TAGs under different nutrient starvation conditions. The total lipid was estimated using phosphovanillin assay (Figures 2A \& 2B) and fatty acid methyl esters (FAMEs) also known to be neutral lipid were quantitatively determined by GC-MS (Figures 3A \& 3B). Also our data has shown relation between these methods that can be reproducible i.e. GC-MS derived quantitative values will be $\sim 3$ times more than that of phosphovanillin assay (Figures 2A \& 2B). In Chlorella sp. the neutral lipid content seems to be drastically enhancing under nutrient deprivation although FAME productivity was higher in nitrogen $(-\mathrm{N})$ and sulfur $(-\mathrm{S})$ starvation than phosphorus (-P) (Figure 3A). In Tetraselmis sp. nutrient limitation has profound effect on accumulation of lipids in all stress conditions (Figure 3B). However, the FAME productivity was higher under nitrogen (-N) starvation in Chlorella sp. $\left(\sim 17.4 \mathrm{mg} \mathrm{L}^{-1} \mathrm{D}^{-1}\right)$ whereas Tetraselmis sp. showed highest FAME productivity in phosphorus starvation $(\sim 16$ $\mathrm{mg} \mathrm{L}^{-1} \mathrm{D}^{-1}$ ) at the end of day 6 . The highest productivity under phosphorus (-P) deprivation in case of Tetraselmis sp. can be attributed to its increased growth as well as lipid accumulation with compared to Chlorella sp. which did not accumulate much lipid under phosphorus (-P) deprivation. Thus, this phenomenon can be an alternative approach to enhance lipid content among specific microalgal strains without compromising growth. In both these strains, nitrogen starvation had a severe effect on growth but also lead to increase in neutral lipid production by onset of nutrient depletion ( $2^{\text {nd }}$ day) (Figures $3 \mathrm{~A} \& 3 \mathrm{~B}$ ). This demonstrates that most of the metabolic responses and biochemical changes initiates immediately upon nutrient deprivation. 

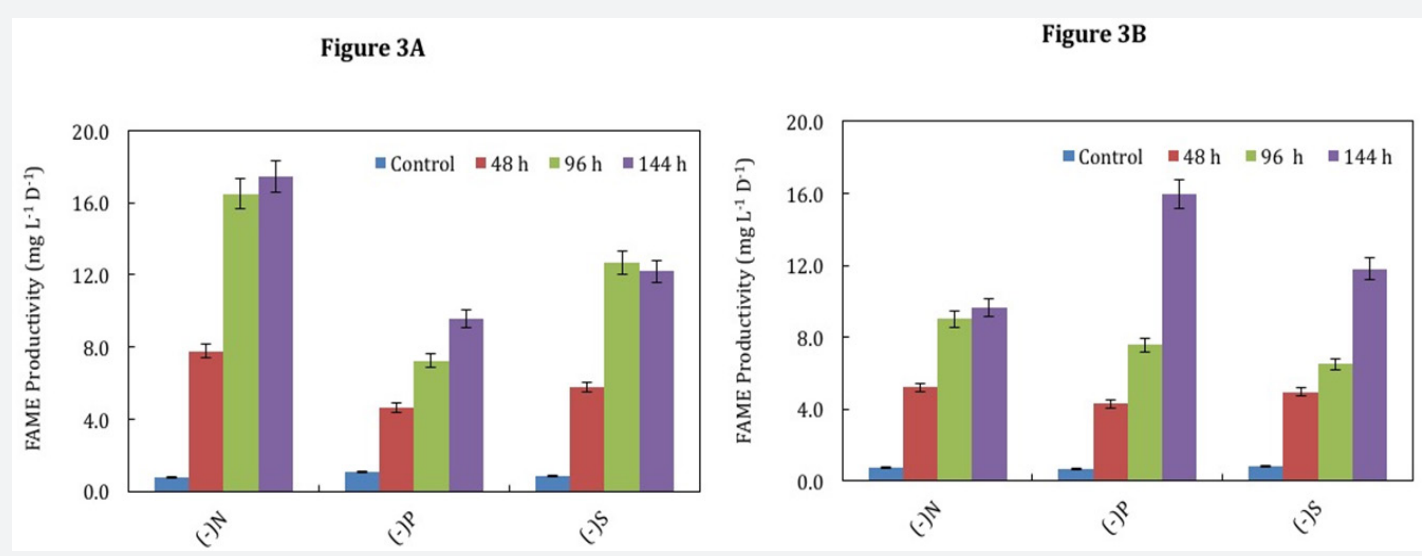

Figure 3A: FAME productivity in freshwater microalgae Chlorella sp. subjected to $0,48,96$, and $144 \mathrm{~h}$ of treatment under nutrient deplete $[-\mathrm{N} ;-\mathrm{P} ;-\mathrm{S}]$ conditions with continuous illumination of light in minimal (BG-11) media.

Figure 3B: FAME productivity in freshwater microalgae Tetraselmis sp. Subjected to 0,48, 96, and 144h of treatment under nutrient deplete $[-\mathrm{N} ;-\mathrm{P} ;-\mathrm{S}]$ conditions with continuous illumination of light in minimal (BG-11) media

Nitrogen is critical for protein synthesis, but under limiting conditions most of the carbon fixed in photosynthesis is used for producing lipid or carbohydrates, instead of proteins $[23,29,32$ 35]. Thus, nitrogen is considered most important nutrient affecting lipid metabolism in microalgae. sulfur is one of the significant nutrients that affect the biohydrogen production in microalgae $[6,33]$. Our studies have also shown that sulfur deprivation also led to increased total lipid content in the Chlorella sp. and Tetraselmis sp. Phosphorous is involved in many cellular metabolic processes and results in accumulation of lipids. In Scenedesmus sp LX1, lipid accumulates up to 53\% under phosphorus-limited conditions [36,37]. Consequently, thus desired biofuels precursors can be achieved by manipulating nutrient conditions for enhanced production of sustainable renewables.

\section{Conclusion}

In the present study, two oleaginous microalgae Chlorella sp. and Tetraselmis sp. were assessed for understanding the stress biology that can simultaneously produce lipids and other high value precursors useful for different applications in the areas of bioenergy including others such as pharmaceutical and nutraceutical industries. Nutrient limitation in both these strains led to a severe growth arrest, with few exceptions under phosphorous-limiting. Nitrogen $(-\mathrm{N})$, phosphorus $(-\mathrm{P})$, and sulfur $(-S)$ starvation increased the lipid content in both freshwater strains. Lipid profiling shows a recycling of lipids and change in the saturation and instauration level of fatty acids under different nutrient-limiting conditions. Nitrogen limitation increased the lipid content to higher levels and the saturation and instauration level were found to be favourable for biofuel precursors. In conclusion, the biomass and lipid productivity among these freshwater strains seems to be competitive, and further characterization may be a critical step towards making these algae-derived biofuels economically competitive for industrial production. With these desirable properties, Chlorella sp. and Tetraselmis sp. would be highly suitable candidates for simultaneous production of lipids and biorenewables ( $\mathrm{B}^{3} \sim$ Biomass, Biofuels and Biorenewables) without compromising growth. Understanding the entire system through integrated omics research will lead to identify relevant enzyme-encoding genes, and reconstruct the metabolic pathways involved in the biosynthesis and degradation of precursor molecules that may have potential for biofuel production, aiming towards the vision of tomorrow's bioenergy needs.

\section{Acknowledgment}

This work was supported by grants from Department of Biotechnology, Ministry of Science and Technology, New Delhi, India.

\section{References}

1. Qin S, Lin H, Jiang P (2012) Advances in genetic engineering of marine algae. Biotechnol Adv 30(6): 1602-1613.

2. Jutur PP, Asha AN, Se Kwon K (2015) Marine Microalgae: Exploring the systems through an omics approach for biofuel production. Marine Bioenergy: CRC Press, USA, pp. 149-162.

3. Lim DKY, Garg S, Timmins M, Zhang ESB, Thomas Hall SR, et al. (2012) Isolation and evaluation of oil-producing microalgae from subtropical coastal and brackish waters. PLoS One 7(7): e40751.

4. Gong Y, Jiang M (2011) Biodiesel production with microalgae as feedstock: from strains to biodiesel. Biotechnol Lett 33(7): 1269-1284.

5. Paul Abishek M, Patel J, Prem Rajan A (2014) Algae oil: A sustainable renewable fuel of future. Biotechnol Res Int 2014: 272814.

6. Cheng D, He Q (2014) Assessment of environmental stresses for enhanced microalgal biofuel production-an overview. Frontiers in Energy Research.

7. Amaro HM, Guedes AC, Malcata FX (2011) Advances and perspectives in using microalgae to produce biodiesel. Applied Energy 88(10): 3402-3410.

8. Cao J, Yuan H, Li B, Yang J (2014) Significance evaluation of the effects of environmental factors on the lipid accumulation of Chlorella minutissima UTEX 2341 under low-nutrition heterotrophic condition. Bioresour Technol 152: 177-184. 
9. Demirbas A, Fatih Demirbas M (2011) Importance of algae oil as a source of biodiesel. Energy Conversion and Management 52(1): 163170.

10. Mata TM, Martins AA, Caetano NS (2010) Microalgae for biodiesel production and other applications: A review. Renewable and Sustainable Energy Reviews 14(1): 217-232.

11. Singh B, Guldhe A, Rawat I, Bux F (2014) Towards a sustainable approach for development of biodiesel from plant and microalgae. Renewable and Sustainable Energy Reviews 29: 216-245.

12. Talebi AF, Tohidfar M, Tabatabaei M, Bagheri A, Mohsenpor M, et al. (2013) Genetic manipulation, a feasible tool to enhance unique characteristic of Chlorella vulgaris as a feedstock for biodiesel production. Mol Biol Rep 40(7): 4421-4428.

13. Sharma KK, Schuhmann H, Schenk PM (2012) High Lipid induction in microalgae for biodiesel production. Energies 5: 1532-1553.

14. Selvarajan R, Felföldi T, Tauber T, Sanniyasi E, Sibanda T, et al. (2015) Screening and evaluation of some green algal strains (Chlorophyceae) isolated from freshwater and soda lakes for biofuel production. Energies 8(7): 7502-7521.

15. Duong VT, Li Y, Nowak E, Schenk PM (2012) Microalgae: Isolation and selection for prospective biodiesel production. Energies 5(6): 18351849.

16. Minhas Ak, Hodgson P, Barrow C, Adholeya A (2016) A review on the assessment of stress conditions for simultaneous production of microalgal lipids and carotenoids. Front Microbiol 7: 546.

17. Harris EH (2009) The Chlamydomonas source book: Introduction to Chlamydomon as and its laboratory use: Elsevier Science.

18. Mishra SK, Suh WI, Farooq W, Moon M, Shrivastav A, et al. (2014) Rapid quantification of microalgal lipids in aqueous medium by a simple colorimetric method. Bioresour Technol 155: 330-333.

19. Higgins BT, Thornton Dunwoody A, Labavitch JM, Vander Gheynst JS (2014) Microplate assay for quantitation of neutral lipids in extracts from microalgae. Anal Biochem 465: 81-89.

20. Duong VT, Thomas Hall SR, Schenk PM (2015) Growth and lipid accumulation of microalgae from fluctuating brackish and sea water locations in South East Queensland-Australia. Front Plant Sci 6: 359.

21. Brown AP, Slabas AR, Rafferty JB, Wada H, Murata N, et al. (2010) Fatty acid biosynthesis in plants - Metabolic pathways, structure and organization. Lipids in Photosynthesis: Essential and Regulatory Functions. Dordrecht: Springer Netherlands, pp. 11-34.

22. Chen M, Tang H, Ma H, Holland TC, Ng KYS, et al. (2011) Effect of nutrients on growth and lipid accumulation in the green algae Dunaliella tertiolecta. BioresourTechnol 102(2): 1649-1655.

23. Gao Y, Yang M, Wang C (2013) Nutrient deprivation enhances lipid content in marine microalgae. Bioresour Technol 147: 484-491.

24. Wang ZT, Ullrich N, Joo S, Waffenschmidt S, Goodenough U, et al. (2009) Algal lipid bodies: stress induction, purification, and biochemical characterization in wild-type and starchless Chlamydomonas reinhardtii. Eukaryotic Cell 8(12): 1856-1868.

25. Valenzuela J, Carlson RP, Gerlach R, Cooksey K, Peyton BM, et al (2013) Nutrient resupplementation arrests bio-oil accumulation in Phaeodactylum tricornutum. Appl Microbiol Biotechnol 97(15): 70497059.

26. Gordon JM, Polle JEW (2007) Ultrahigh bioproductivity from algae. Appl Microbiol Biotechnol 76(5): 969-975.

27. Fields MW, Hise A, Lohman EJ, Bell T, Gardner RD, et al. (2014) Sources and resources: importance of nutrients, resource allocation, and ecology in microalgal cultivation for lipid accumulation. Appl Microbiol Biotechnol 98(11): 4805-4816.

28. Hu Q, Sommerfeld M, Jarvis E, Ghirardi M, Posewitz M, et al. (2008) Microalgal triacylglycerols as feedstocks for biofuel production: perspectives and advances. Plant J 54(4): 621-639.

29. Msanne J, Xu D, Konda AR, Casas Mollano JA, Awada T, et al. (2012) Metabolic and gene expression changes triggered by nitrogen deprivation in the photoautotrophically grown microalgae Chlamydomonas reinhardtii and Coccomyxa sp C-169. Phytochemistry 75: 50-59.

30. Markou G, Nerantzis E (2013) Microalgae for high-value compounds and biofuels production: a review with focus on cultivation under stress conditions. Biotechnology Advances 31(8): 1532-1542.

31. Halsey KH, O’Malley RT, Graff JR, Milligan AJ, Behrenfeld MJ (2013) A common partitioning strategy for photosynthetic products in evolutionarily distinct phytoplankton species. New Phytol 198(4): 1030-1038.

32. Philipps G, Happe T, Hemschemeier A (2012) Nitrogen deprivation results in photosynthetic hydrogen production in Chlamydomonas reinhardtii. Planta 235(4): 729-745.

33. Cakmak T, Angun P, Demiray YE, Ozkan AD, Elibol Z, et al. (2012) Differential effects of nitrogen and sulfur deprivation on growth and biodiesel feedstock production of Chlamydomonas reinhardtii. Biotechnol Bioeng 109(8): 1947-1957.

34. Juergens MT, Deshpande RR, Lucker BF, Park JJ, Wang H, et al. (2015) The regulation of photosynthetic structure and function during nitrogen deprivation in Chlamydomonas reinhardtii. Plant Physiology 167(2): 558-573

35. Miller R, Wu G, Deshpande RR, Vieler A, Gartner K, et al. (2010) Changes in transcript abundance in Chlamydomonas reinhardtii following nitrogen deprivation predict diversion of metabolism. Plant Physiol 154(4): 1737-1752.

36. Xin L, Hu HY, Ke G, Sun YX (2010) Effects of different nitrogen and phosphorus concentrations on the growth, nutrient uptake, and lipid accumulation of a freshwater microalga Scenedesmus sp. Bioresource Technology 101(14): 5494-5500.

37. Chu FF, Chu PN, Shen XF, Lam PK, Zeng RJ, et al. (2014) Effect of phosphorus on biodiesel production from Scenedesmus obliquus under nitrogen-deficiency stress. Bioresour Technol 152: 241-246. 


\section{Your next submission with Juniper Publishers will reach you the below assets}

- Quality Editorial service

- Swift Peer Review

- Reprints availability

- E-prints Service

- Manuscript Podcast for convenient understanding

- Global attainment for your research

- Manuscript accessibility in different formats

( Pdf, E-pub, Full Text, Audio)

- Unceasing customer service

Track the below URL for one-step submission https://juniperpublishers.com/online-submission.php 